\title{
Node-Gosper Island-Based Data Collection Method with Recharge Plan for Rechargeable Wireless Sensor Networks
}

\author{
Chi-Chang Chen $^{l *}$ \\ ${ }^{1}$ Department of Information Engineering, I-Shou University, 84001 Kaohsiung, Taiwan
}

\begin{abstract}
Because of recent advances in wireless power transfer technologies, several key topics of wireless sensor networks (WSNs) need to be revisited. Traditional data collection methods for WSNs typically consider the balance of power consumption among sensors as a critical design criterion for avoiding uneven power depletion in the networks. I propose a solution for the uneven power consumption problem of data collection over WSNs. I designed a node-Gosper island-based scalable hierarchical cluster transmission method in conjunction with a wireless recharge plan for data collection over rechargeable WSNs. For the recharge plan, I used a mobile wireless charger to visit and recharge the batteries of sensors located on different levels of node-Gosper curves with various frequencies. The duration of each recharging process for each layer of sensors was calculated to verify the feasibility of the proposed recharge plan. The simulation results indicate that my proposed data collection method outperforms several well-known data collection methods in terms of energy consumption.
\end{abstract}

\section{Introduction}

A wireless sensor network (WSN) is a wireless communication network consisting of numerous automated devices that use sensors to cooperate in monitoring the physical or environmental conditions of designated locations, such as temperature, sound, vibration, pressure, movement, and contaminants. In recent years, the number of studies on WSNs has substantially increased. In such studies, the method of extending the lifetime of WSNs is always the key topic considered for designing protocols or algorithms for WSNs. Although previous efforts have provided several effective energy-saving methods, the ability to extend the lifetime of WSNs is still limited by the battery power configured in the sensors.

Wireless charging technology originated approximately 100 years ago with American scientist Nikola Tesla. In 2007, at the Massachusetts Institute of Technology (MIT), a group of theoretical physicists performed an electromagnetic resonance power transmission of $2 \mathrm{~m}$ to light a $60-\mathrm{W}$ bulb [1], thereby bringing wireless power transmission into a new era.

In this work, I propose an energy-efficient data collection method for a rechargeable WSN (RWSN) based on the recursive structures of node-Gosper islands. In association with the proposed data collection method, I provide a novel recharge plan that uses a wireless charger travelling in different levels of node-Gosper curves to charge the sensors in the RWSN by using electromagnetic resonance power transmission technology. I simulated my proposed data collection method and compared the performance with several well-known data collection methods. The simulation results indicate that my proposed method outperforms those well-known data collection methods in terms of energy consumption.

\section{Background and related works}

A node-Gosper curve is a space-filling curve of fractal geometry generated by the continuous replacement of each vertex with the basic seven-segment curve. The resultant filled space is a node-Gosper island [2]. Figure 1 shows level-1 to level-3 node-Gopser islands, respectively, with their corresponding node-Gosper curves. Recently, the node-Gosper curve has been successfully applied to solve the mobile anchor localization problems of WSNs [3]. In the literature, a variety of hexagonal addressing methods for identifying hexagons in a hexagonal tessellated plane are described. In the present study, I used two addressing methods, namely the ring-based addressing scheme and base-7 addressing scheme, described in [3], both of which are suitable for addressing the hexagons in a node-Gosper island.

Generally, the data transmission methods of WSNs can be divided into three categories: direct transmission, multihop transmission, and cluster transmission. Direct transmission involves each sensor collecting and directly transmitting data to the base station. It is the simplest data transmission method. For multihop transmission, each sensor transmits data to neighboring nodes that are closer to the base station. This routing method can avoid mass energy consumption for the sensors that are far away from the base station. However, because data from 

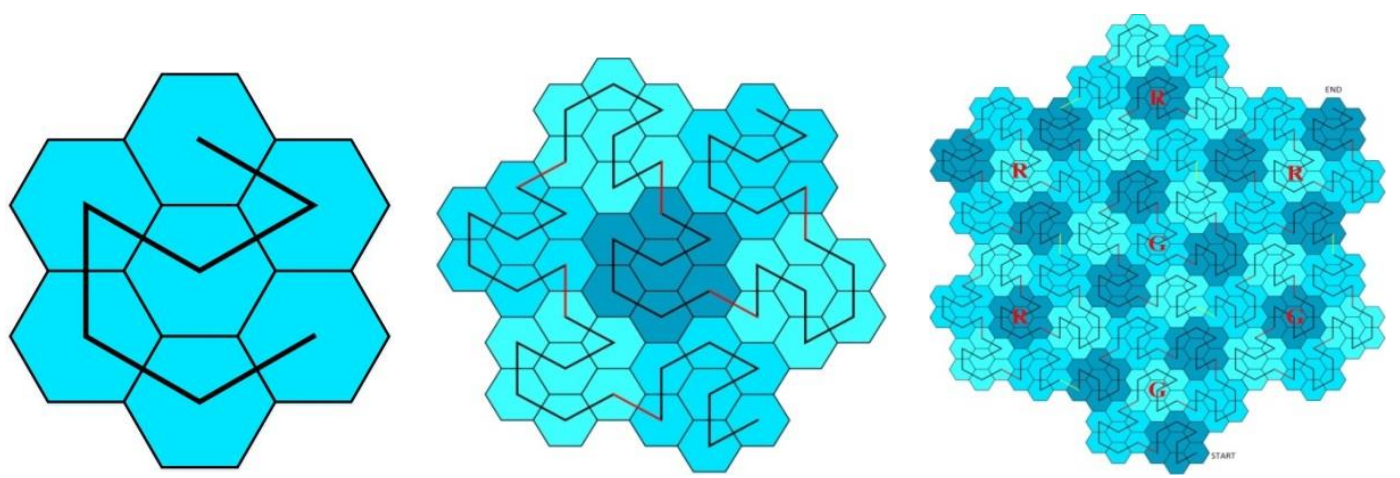

Fig. 1. Level-1, level-2, and level-3 node-Gosper islands and their corresponding node-Gosper curves.

all sensors are continuously transmitted to the base station, those that are close to the base station must transfer more information, resulting in the energy of these sensors being exhausted faster than that of others. Clustering transmission serves as a compromise between the previous two methods. First, the entire sensor network is divided into several clusters according to a specific topological relationship. Each cluster periodically selects its cluster head according to a protocol, and then all the sensors in the cluster transmit their collected data to the respective cluster head. Finally, the cluster head sends the aggregated data to the base station in a direct or multihop manner. Among all cluster transmission methods, the low-energy adoptive cluster hierarchy (LEACH) [4] is the most well-established.

Recent data collection methods for RWSNs, such as those proposed in [5], [6] and [7], usually performed wireless charging and data gathering functions over the same path for the same duration. However, for general WSNs, the durations for charging sensors and collecting data are typically different. The duration for data collection is typically measured in seconds and minutes and is much faster than that of charging the sensors in the network (possibly measured in months or years). Therefore, in this study, I separated these two functions, and performed them with different paths and different durations.

\section{Proposed data collection method and recharge plan}

In this section, I focused on the data collection methods of the RWSN and proposed a new method that uses the energy of the whole RWSN more efficiently. Additionally, in association with the data collection method, I provided an efficient recharge plan to charge all the sensors in the RWSN. I assumed that the RWSN contains two types of sensors, namely relay sensors and normal sensors, and that the base station was located at the center of the sensing area. Each relay sensor was installed with the long-range Zigbee communication chip, the communication range of which is over $1 \mathrm{~km}$ [8]. Each normal sensor was installed with the normal Zigbee communication chip, the communication range of which is $10-60 \mathrm{~m}$ depending on the environment of the sensing field. Both types of sensors were assumed to have the same amount of initial energy.

\subsection{Proposed data collection method for the RWSN}

In this subsection, I assume that the entire sensing area of the RWSN was covered by a sufficient level of the node-Gosper island and a relay sensor was deployed at the center of each sub-node-Gosper island. In my proposed data collection method, I adopted the hierarchical clustering approach based on the recursive structure of the node-Gosper island. I considered every seven basic hexagons (i.e., the first level node-Gosper island) as a basic cluster. Each sensor transmits its collected data to the center of the central hexagon in its cluster. In order to allow each normal sensor to send its collected data to its cluster head, the radius of the circumscribed circle of each basic hexagon is set to $r / 3$, where $r$ is the communication range of the normal sensors in the RWSN. Because the node-Gosper island has a recursive structure, the higher level node-Gosper island is composed of seven lower level sub-nodeGopser islands. Therefore, the sensor data can be transmitted to the central hexagon of the higher level node-Gosper island using the same method until all the data have been passed to the center of the central hexagon of the entire sensor network.

The proposed data collection method is summarized as the following two stages:

1) Setup Stage:

1. Cover the entire sensing area with the minimal level, say $\mathrm{k}$, node-Gosper island, in which the radius of the circumscribed circle of each hexagon is set to $\frac{r}{3}$, where $r$ is the communication range of the normal sensors in the RWSN.

2. Assign a base-7 address to each hexagon in the level-k node-Gosper island and calculate the Cartesian coordinates of the center of each addressed hexagon. Derive the node-Gosper curve of the level-k node-Gosper island by using the base7 addressing sequence. 

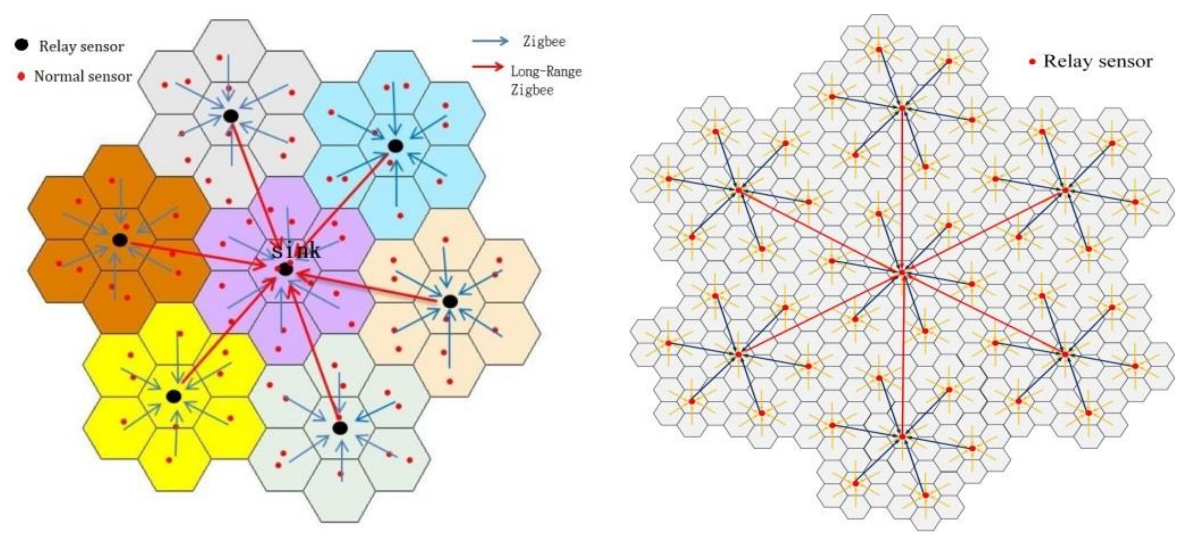

Fig. 2. Data collection paths in the level-2 and level-3 node-Gosper islands.

3. Localize all the sensors and separately identify the hexagons that they belong to. This can be achieved by programming a robot car equipped with a wireless communication chip and a GPS device to travel along the level-k node-Gosper curve obtained in Step 2. When the robot car reaches a turning point, it broadcasts its position message to all sensors within its communication range. Once a sensor has received more than three different position messages from the robot car, that sensor can estimate its coordinates by using the trilateration method, and subsequently identify the hexagon that the sensor belongs to based on the estimated coordinates.

4. A relay sensor is deployed at the center of each level-1 sub-node-Gosper island in the level-k nodeGosper island, either manually or by using a robot car (an example of a deployed level-2 node-Gosper island is shown in the left side of Figure 2).

\section{2) Data Transmission Stage:}

1. Each normal sensor sends its data to the relay sensor located at the center of the level-1 sub-node-Gosper island that the normal sensor belongs to based on the address of the hexagon it located.

2. Each relay sensor in the level-1 sub-node-Gosper island aggregates the collected data and sends the aggregated data to the relay sensor of the level-2 sub-node-Gosper island that it belongs to. Similarly, if applicable, each relay sensor of a lower level subnode-Gosper island sends its collected data to the relay sensor of its immediate higher level sub-nodeGosper island until the base station located at the center of the level-k node-Gosper island is reached (Fig. 2 depicts the data collection paths of the level2 and level-3 node-Gosper islands, respectively).

If the aforementioned method is used to transfer the collected data to the base station, the relay sensors of the higher level sub-node-Gosper islands must send more information with longer transmission distances, causing their power consumption levels to increase to levels higher than those of the lower level relay sensors. This phenomenon is restricted by the traditional data collection method in a WSN because the relay sensors of higher level sub-node-Gosper islands become invalid much earlier than do other sensors.

In the next subsection, I consider the aforementioned phenomenon and provide details of charging cycles for different sensors with different levels of power consumption to ensure that the power supply to all sensors in the sensor networks is sufficient and stable.

\subsection{Recharge plan for the sensors in the RWSN}

In this study, I outline the use of a wireless mobile charger called ChargerCar to charge the sensors in an RWSN. ChargerCar is a robot car equipped with a highcapacity battery, magnetic resonance power transmitter, wireless communication transceiver, and GPS.

According to the data collection method proposed in the previous subsection, I assumed that the sensing region of the RWSN is covered by a level-k nodeGosper island. The most power-consuming nodes are the relay sensors located at the center of the surrounding six level-(k-1) sub-node-Gosper islands, which I call layer1 relay sensors. Considering the level-3 node-Gosper island in Fig. 8 as an example, the most energyconsuming nodes are the six layer-1 relay sensors located at the centers of the six level-2 sub-node-Gosper islands. Because I assumed that all the sensors in the RWSN have the same initial energy, the most energyconsuming sensors should be recharged earlier than the other sensors. For this purpose, we can use the path of the level-1 node-Gosper curve depicted in Fig. 9 as the routing path for ChargerCar to charge the layer-1 relay sensors located at the centers of the level-2 sub-nodeGosper islands.

Subsequently, the relay sensors in each level-(k-2) sub-node-Gosper island called layer-2 relay sensors can be arranged as the next charging nodes by using the level-2 node-Gosper curve. Fig. 10 illustrates the level-3 node-Gosper island and corresponding level-2 sub-nodeGosper curve as the charge path for layer-2 relay sensors. Similarly, for the other relay sensors in level- $(k-i), i=$ $3, . .,(\mathrm{k}-1)$ sub-node-Gosper islands, called layer- $i$ relay 


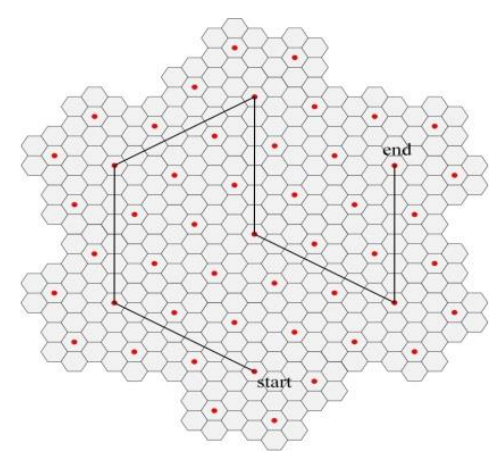

(a)

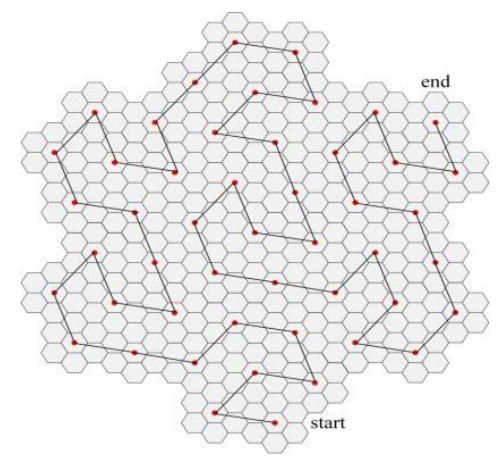

(b)

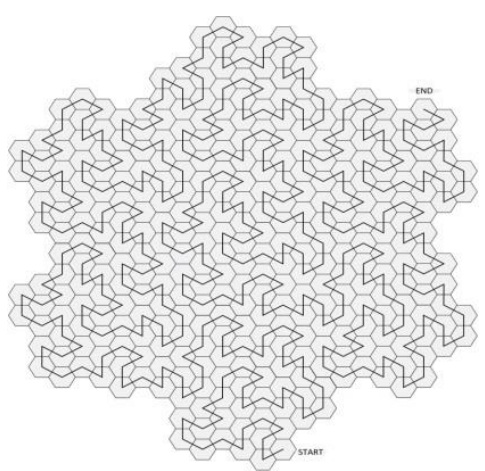

(c)

Fig. 3. Charge path of (a) layer1 relay sensors, (b) layer-2 relay sensors, and (c) normal sensors.

sensors, the node-Gosper curve of level- $i$ can be used as the charge path for the relay sensors.

Finally, the charging path for all the normal sensors in the RWSN can follow the level-k node-Gosper curve. As shown in Fig. 11, ChargerCar can stay at the center of each hexagon to charge the sensors in a one-to-many manner in the hexagon. Because each normal sensor in the data collection method mentioned in the previous subsection transmits the sensing data directly and over a short distance to the relay sensor at the center of the level-1 sub-node-Gosper island to which it belongs, the power consumption is very low. Therefore, the energy of each normal sensor can last for an extended period before needing to be recharged.

If the sensing area is covered by a level-k nodeGosper island, the charge plan for the RWSN can be arranged as follows:

1. Estimate the cycles of recharge time for the relay sensors on each layer and the normal sensors according to the amount of data they have transmitted and received. Suppose that the cycles of recharge time for the relay sensors on layer- $i$ are $R_{i}$ days, where $i=1,2, \ldots, \mathrm{k}-1$, and for the normal sensors is $N$ days.

2. For each $i$ from 1 to $\mathrm{k}-1$, identify the centers of the $7^{i} \quad$ level-(k-i) sub-node-Gosper islands that constitute the level-k node-Gosper island. Every $R_{i}$ days, send ChargerCar to visit and charge the layer- $i$ sensors located at the $7^{i}$ center positions along the path of the level- $i$ node-Gosper curve.

Every $N$ days, send ChargerCar to travel along the level-k node-Gosper curve and stop at the center of each hexagon (i.e., the turning point of the node-Gosper curve) to charge the normal sensors in the hexagon in the oneto-many manner.

\section{Simulation results}

In this section, I simulate my proposed data collection method and compare its performance of various types of energy consumption with those of three other well-known data collection methods, namely direct transmission, the LEACH [4], and multihop routing with minimum hop counts [9].
The energy consumption model from the LEACH was applied to all sensors as follows:

$$
\begin{aligned}
& E_{T X}(k, d)= \begin{cases}k E_{\text {elec }}+k \varepsilon_{f s} d^{2}, & d<d_{0} \\
k E_{\text {elec }}+k \varepsilon_{m p} d^{4}, & d \geq d_{0}\end{cases} \\
& E_{R X}(k)=E_{\text {elec }} \times k
\end{aligned}
$$

where $E_{T X}$ and $E_{R X}$ are the energy consumed when transmitting and receiving, respectively, $\mathrm{k}$ bits data from a source node to the anchor node. The sensors in the cluster used TDMA as the medium access control layer control protocol for communication. The simulation tool used was MATLAB R2013a. Simulations of 50 different deployments of sensors were performed and the mean values were taken for comparison. In the following, I showed the simulation results of different type of energy consumptions for my proposed method (node-Gosper) with aforementioned three well-known methods.

\subsection{Accumulated energy consumption of all sensors}

Figure 4(a) illustrates the simulation results of the accumulated energy consumption for the four data collection methods with 200 sensors. The figure indicates that my proposed method (node-Gosper) is superior to the direct transmission method, multihop routing with minimum hop count method, and LEACH. Fig. In the simulation, sensors are randomly distributed in the sensing field. Thus, many sensor nodes may locate far away from the base station. According to Equation (1) of the energy consumption model, when the transmission distance is greater than $87.7 \mathrm{~m}$ (i.e., $d_{0}$ ) [4], the transmission energy consumption is proportional to the fourth power of its distance. Therefore, if the direct transmission method is applied, these sensors consume very large amounts of energy and increase the total energy consumption to a very high level.

Regarding the LEACH method, a Voronoi diagram is used for clustering. Because the clusters vary in size, some of the sensor nodes may have communication distances greater than $87.7 \mathrm{~m}$, despite data being sent to their cluster heads within their clusters. Additionally, the election of a cluster head for each round consumes a certain amount of energy from all sensors. These factors 


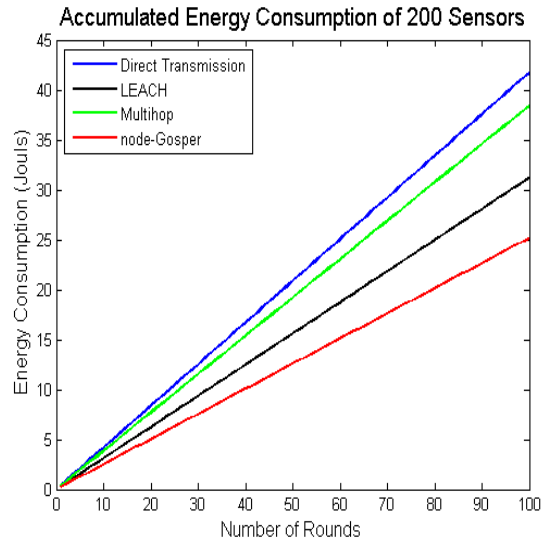

(a)

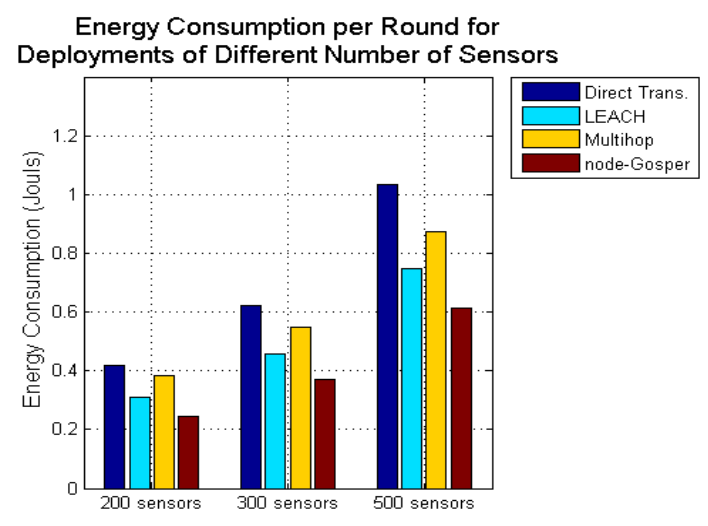

(b)

Fig. 4. (a) Comparison of the accumulated energy consumption of 200 sensors for the four data collection methods. (b) Comparison of the average energy consumption per round for different number of sensors deployed in the sensing area for the four data collation methods

render the total energy consumption of the LEACH higher than that of my proposed method.

Regarding multihop routing with minimum hop count method, because sensors do not cluster, no data aggregation effect occurs. Although the distance between each hop is shorter, the total energy consumption of all sensors is still higher than that of my proposed method.

In my proposed method, the cluster size and the locations of cluster heads are fixed. Except for the lower layer relay sensors, almost all the transmissions between sensors occur over a short distance, and this conserves energy. Because my approach causes the lower layer relay sensors to consume excessive amounts of energy, this approach may not be suitable for traditional WSNs because of the uneven power consumption of sensors. However, in the RWSN, this problem can be resolved by shortening the recharging cycle for the lower layer relay sensors to achieve a sufficient energy provision environment.

\subsection{Energy consumption for different node- densities}

In this subsection, different number of sensors is deployed in the sensing area of each data collection method. I compared the average energy consumption of the sensors per round for the deployment of 200,300 , and 500 sensors in the same area of each method. The simulation result is shown in Figure 4(b), which indicates that my proposed method (node-Gosper) consumed less energy than the other three data collection methods for all the cases of different number of sensor deployments.

In my proposed method, as long as the topology of node-Gosper area is fixed, the number of relay sensors is fixed regardless the increase of the number of sensors. Therefore, as the number of sensors increases, the additional sensors are all normal sensors, which transmit sensing data in a very short distance, and they consume very little amount of energy. Due to the benefit of the data aggregation in the cluster head, the relay sensors only need to transmit $60 \%$ (the assumption in the simulation) of the data amount to their upper level. Therefore, the amount of energy consumption is less than the direct transmission method and multihop method, which do not have the clustering effect. For the LEACH method, the number of cluster heads $(5 \%$ of the sensors in this experiment) increases as the number of sensors increases. Thus, there may be more cluster heads which have distance greater than $87.7 \mathrm{~m}$, and these cluster heads consume a large amount of energy while they transmit collected data to the base station.

\subsection{Average energy consumption of each sensor}

In this subsection, I compare the average energy consumption of each sensor in the four data collection methods. In particular, I focus on the comparison of the normal sensor in my proposed method (node-Gosper) with the sensors in the other methods. In the simulation, I measured the average energy consumption of all the sensors. In my proposed method, I measured energy consumption of two types of sensors, namely relay sensors and normal sensors. Figure 5 indicates that the average energy consumption of the normal sensors in my method is much less than that of the sensors in the other methods. The reason is that the normal sensors, which the majority of the sensors, in my proposed method only need to send their sensing data to their corresponding cluster heads, and these transmissions are all within a short distance. On the other hand, most of the sensors in the multihop method not only need to send their sensing data, but also need to relay data for other sensors. In the LEACH method, although the non-cluster head sensors only need to send the sensing data for themselves, they may locate at a considerable distance to their cluster head due to the large size of the clusters.

As explained in the first subsection, the lower layer relay sensors in my proposed method may consume excessive amounts of energy. This problem can be resolved by shortening the recharging cycle for the lower 
Table 1. Path lengths and number of stop stations required to charge all sensors in the RWSN

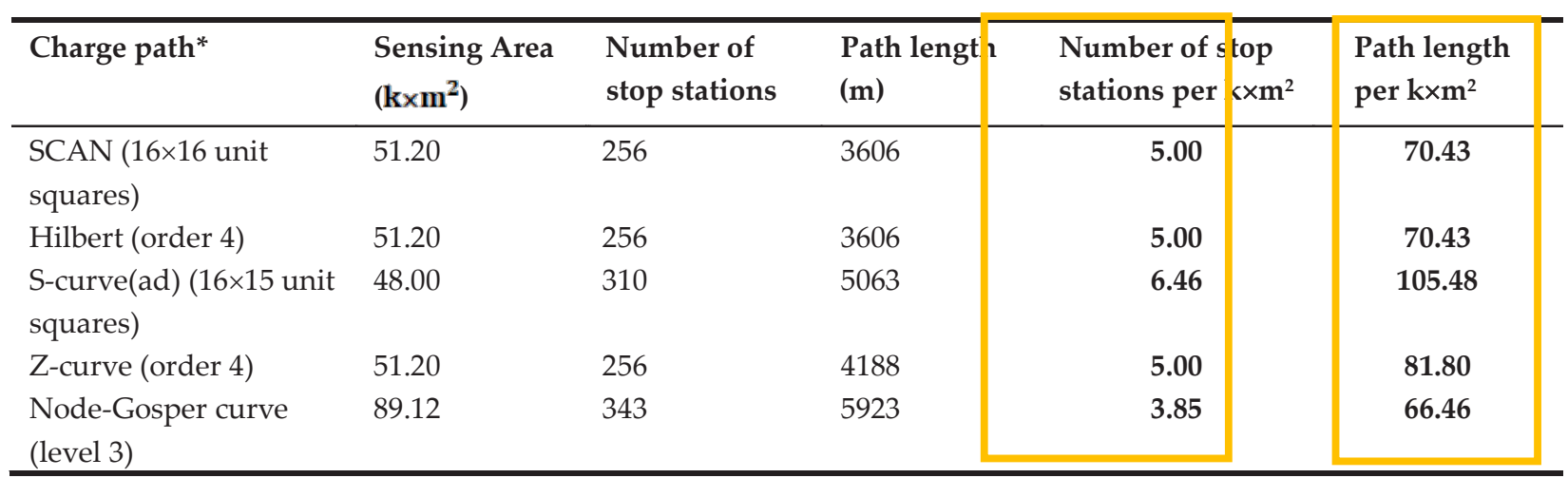

* The radius of the circumscribed circle of each unit square (or hexagon) was $10 \mathrm{~m}$.

layer relay sensors to achieve a sufficient energy provision environment in the RWSN.

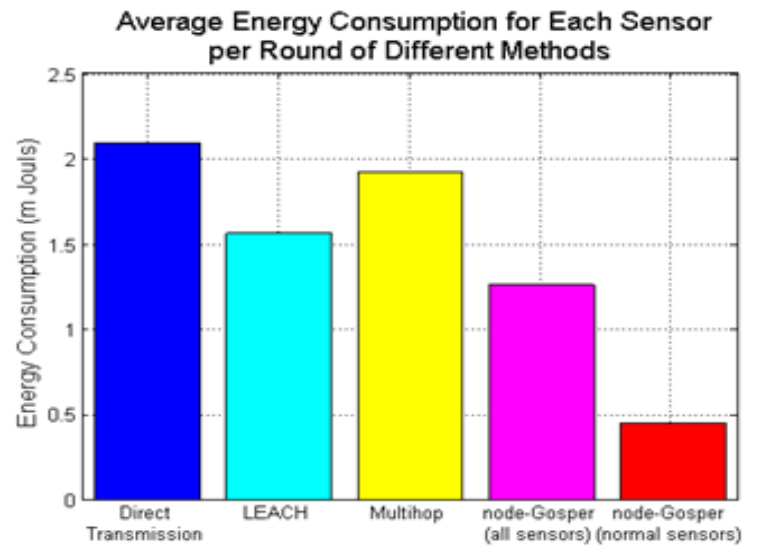

Fig. 5. Comparison of energy consumption of each sensor for the four data collection methods.

\subsection{Comparison of various charge paths in the recharge plans}

In [10] Han et al. used four well-known routing paths from traditional WSNs, namely the SCAN, Hilbert curve, S-curve(ad), and Z-curve, as charge paths for the RWSN. Table 1 compares the path lengths and number of stop stations of the node-Gosper curve, SCAN, Hilbert curve, S-curve(ad), and Z-curve, assuming that each unit regular polygon (square or hexagon) has the same radius $(\mathrm{r}=10 \mathrm{~m})$ of the circumscribed cycle. My proposed method has less number of stop stations and shorter path travel path than other charge paths over the same normalized area.

\section{Conclusions}

This paper presents a novel data collection method with a recharge plan for RWSNs. My method is based on the node-Gosper island in association with its corresponding node-Gosper curves. I compared my proposed method with three well-known data collection methods, namely direct transmission, the LEACH, and multihop routing with minimum hop count. My proposed method outperformed these three traditional data collection methods in terms of many respect of energy consumption, including the accumulated energy consumption of sensors, different densities of sensors, and average energy consumption of sensors.

I also compared the charge path lengths and the number of charge stops of the charge paths when separately using the node-Gosper curve, SCAN, Hilbert curve, S-curve(ad), and Z-curve for the RWSNs. The results in Table 1 indicate that the node-Gosper curve had the shortest charge path length and lowest number of charge stops among all the compared curves. This result implies that the node-Gosper curve is a suitable candidate for use as a charge path for RWSNs.

In recent years, wireless power transfer technologies have increased in popularity in many application domains such as electric vehicles and mobile phones. The cost of wireless power transfer modules is predicted to gradually decrease. Therefore, a rechargeable battery module is expected to soon become available for installation on sensor nodes in WSNs. In such a case, many existing solutions for WSNs from network protocols to working applications must be revisited and adjusted accordingly. I believe that more effort should be devoted to this newly derived research direction in the future.

Acknowledgments: This work was partially supported by the Ministry of Science and Technology, Taiwan, under contract MOST106-2221-E-214-010.

\section{References}

1. A. Kurs, A. Karalis, R. Moffatt, J. D. Joannopoulos, P. Fisher, M. Soljačić, Wireless power transfer via strongly coupled magnetic resonances. Science, 317(5834), 83-86 (2007)

2. J. Ventrella, "Root 7 Family." Brainfilling Curves-A Fractal Bestiary. Lulu.com (2012)

3. Chi-Chang Chen, Sheng-Bo Wang, Node-Gosper Curve-Based Unknown Sensor Localization Using Single Mobile Anchor in Wireless Sensor Networks. International Journal of Distributed Sensor Networks, 2016(7),13 pages (2016) 
4. W. R. Heinzelman, A. Chandrakasan, H. Balakrishnan, Energy-efficient communication protocol for wireless microsensor networks. In System sciences, Proceedings of the 33rd annual Hawaii international conference on (pp. 1-10), IEEE (2000)

5. M. Zhao, J. Li, Y. Yang, A framework of joint mobile energy replenishment and data gathering in wireless rechargeable sensor networks. IEEE Transactions on Mobile Computing, 13(12), 26892705 (2014)

6. S. Guo, C. Wang C. Y. Yang, Joint mobile data gathering and energy provisioning in wireless rechargeable sensor networks. IEEE Transactions on Mobile Computing, 13(12), 2836-2852 (2014)

7. B. H. Liu, N. T Nguyen,. V. T. Pham, Y. X. Lin, Novel methods for energy charging and data collection in wireless rechargeable sensor networks. International Journal of Communication Systems (2015)

8. Digi International Inc., XBee/XBee-PRO ${ }^{\circledR} \mathrm{S} 2 \mathrm{C}$ ZigBee ${ }^{\circledR}$ RF Module - Digi International, https://www.digi.com/resources/documentation/digi docs/pdfs/90002002.pdf, accessed on 2017/12/10.

9. I. F. Akyildiz, W. Su, Y. Sankarasubramaniam, E. Cayirci, Wireless sensor networks: a survey. Computer networks, 38(4), 393-422 (2002)

10. G. Han, A. Qian, L. Liu, J. Jiang, C. Zhu, Impacts of traveling paths on energy provisioning for industrial wireless rechargeable sensor networks. Microprocessors and Microsystems, 39(8), 12711278 (2015) 